\title{
Unmanned and autonomous ground vehicle
}

\author{
S. George Fernandez, K. Vijayakumar, R. Palanisamy, K. Selvakumar, D. Karthikeyan, \\ D. Selvabharathi, S. Vidyasagar, V. Kalyanasundhram \\ SRM Institute of Science and Technology, India
}

\begin{tabular}{|c|c|}
\hline Article Info & ABSTRACT \\
\hline $\begin{array}{l}\text { Keywords: } \\
\text { Unmanned and autonomous } \\
\text { ground vehicle } \\
\text { NI vision acquisition system } \\
\text { NI vision development unit }\end{array}$ & $\begin{array}{l}\text { Unmanned and Autonomous Ground Vehicle (UAGV) is a smart vehicle that } \\
\text { capable of doing tasks without the need of human operator. The automated } \\
\text { vehicle can work during off and on road navigation and also used in military } \\
\text { operation such as detecting bombs, border patrol, carrying cargos, search, } \\
\text { rescue etc reducing soldier's exposure to danger, freeing them to perform } \\
\text { other duties. This type of vehicle mainly uses sensors to observe the } \\
\text { environment and automatically take decisions on its own in unpredictable } \\
\text { situation and with unknown information or pass this information to the } \\
\text { operator who control the UAGV through various communication when it } \\
\text { requires support. This UAGV can send visual feedbacks to the operator at the } \\
\text { ground station. An onboard sensor gives the complete environment of the } \\
\text { vehicle as signals to the operator. } \\
\text { Copyright } \odot 2019 \text { Institute of Advanced Engineering and Science. } \\
\text { All rights reserved. }\end{array}$ \\
\hline \multicolumn{2}{|l|}{ Corresponding Author: } \\
\hline \multicolumn{2}{|c|}{$\begin{array}{l}\text { R. Palanisamy, } \\
\text { Department of Electrical and Electronics Engineering, } \\
\text { SRM Institute of Science and Technology, } \\
\text { Kattankulathur, Chennai, India. } \\
\text { Email: krspalani@gmail.com }\end{array}$} \\
\hline
\end{tabular}

\section{INTRODUCTION}

Unmanned ground vehicles (UGV's) are developed for many applications such as the army, in dangerous waste management. Such applications are having limitations on communications which needs navigation autonomously. UGV's have the potential to significantly reduce the critical mistakes that human drivers make. The performance of the UGV's are also better than human drivers because of the better perception and better decision making [1, 2]. The capability of controling an autonomous vehicle is critical which can impact the public acceptance of autonomous vehicles and also the automotive industry [3]. Under these situations, UGV's should be furnished with sensors for spotting obstacles. This paper deals with combination of mechanical, electronics, sensors, image processing and computer vision to make the ground vehicle manual and autonomous enabling the people safety in transportation by eliminating the risk involved in the transportation [4, 5]. The UGV's are embedded with three mobility levels such as teleoperation, computer aided driving and autonomous control. The control station has the capability to simultaneously control the operation and manage UGV's. They are built to handle any missions' missions such as border patrol, surveillance and in active combat both as a standalone unit and as well as a human soldiers (manual). An operator can control comfortably from a remote place wirelessly. This system of ours has two units- one is the control unit (to control mobility) and the other is the motion tracking unit. Both these units have two modes- Automatic and Manual [6]. This robot would be armed with an automatic weapon mounted onto a turret and a remote operator would be getting a live video feed from the camera to help him manually control both the above mentioned units of the rover. The rover is also capable of automatically tracking movement of objects in its range of vision. In the automatic mode, the boat uses Image Processing techniques to track motion. The vehicle has GPS [7, 8] navigation and commands to navigate can be given wirelessly. Additionally, infrared sensors aid in obstacle detection and path mapping. There is one onboard computer, 
which receives command from command center control and issues commands to the onboard microcontroller for controlling the stepper motors, servo motors, wireless data reception, GPS navigation, and obstacle detection [9]. The command center control computer allows the remote user to see the direct video stream and control the various features of the rover, using a GUI [10]. This allows the user to access the vehicle remotely and as well as without hands on the steering and peddle, which is shown in Figure 1.

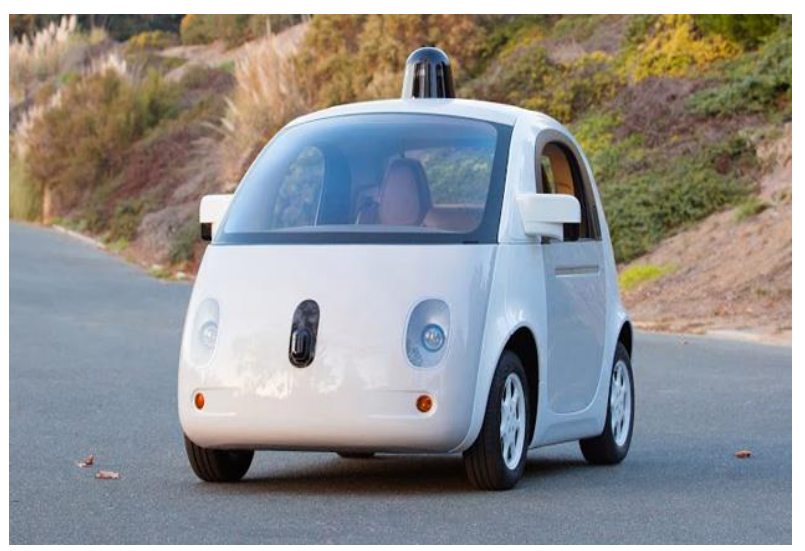

Figure 1. Autonomous car

\section{PROPOSED APPROACH FOR DESIGNING UGV}

The various technologies involved in this UAVG are as follows

- Mechanical design analysing

- Electronics

- Control system

- $\quad$ Image processing

- Computer vision

- Sensor fusion

- Artificial Intelligence

LabView is generally used for data acquisition, instrument control, and industrial automation on a variety of platforms $[11,12]$. Vision software used in LabVIEW has the following components.

- $\quad$ NI Vision Acquisition system

- $\quad$ NI Vision Development Unit

- $\quad$ NI Vision for Automated Inspection

NI Vision Acquisition syatem is the elementary requirement for creating vision kind of applications. The NI Vision software includes the necessary drivers, such as NI - IMAQ and NI - imaqdx. The NI - imaqdx driver software gives you the ability to acquire the image with IEEE 1394 (firewire), Ethernet and Usb cameras. For more advance machine vision image processing you will need NI Vision Development Module. Hundreds of image processing and machine vision functions for labview is available in the Vision development module. This module has pattern matching, texture recognition, counting functions inbuilt [13, 14]. NI Vision Builder is used for automated inspection, gauging, part presence, guidance, and counting. This is external and independent application for building and machine vision application without the need for programming NI visionni Vision Assistant are the tools used in labview.NI Vision is used to create machine vision and scientific imaging applicationwhich helps for basic image analysis, image processing, and machine vision [15]. NI Vision tool helps to create image processing and machine vision applications. Control, and industrial automation on a variety of platforms.

\section{RESEARCH METHOD FOR DESIGN AND CALCULATIONS OF THE PROPOSED MODEL}

This UAGV consists of a camera, ultrasonic sensors, video transmitter and receiver, Zigbee, high torque motors, gps module, digital compass, microprocessor and microcontrollers all combined, the data from all the sensors are collected analysed, fused, processed and finally the limited data is sent to actuators. This combination of all sensors enables the vehicle to take decision in unpredictable and unknown situation to take a right decision to navigate autonomously, Software being used - NI LabVIEW is shown in Figure 2. 


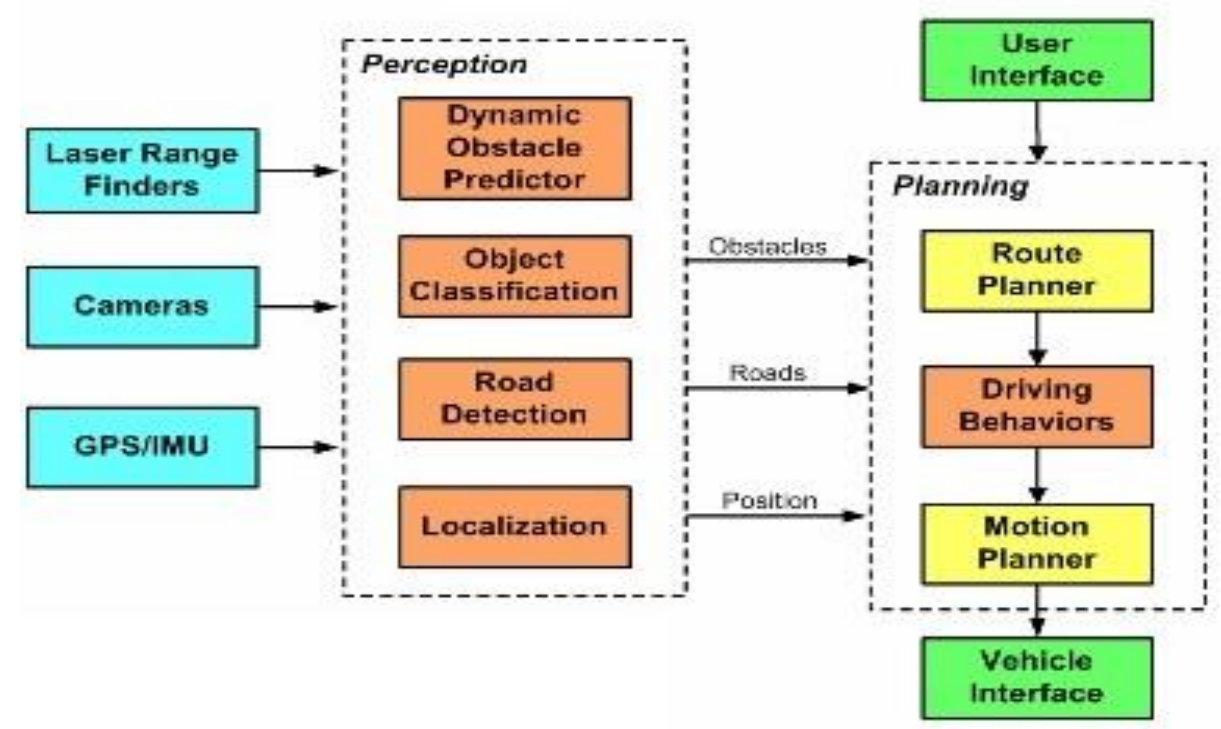

Figure 2. Software being used - NI LabVIEW

The chassis of the vehicle is brought from the outside and analysed the complete mathematical model and the measurements of the vehicle to make a complete redesign in the solid work for virtual prototyping and model in the loop $[16,17]$. This allows checking the control system of the vehicle virtually according to the mechanical design so that the control system is tuned virtually in hit and trailing method with approximate parameters from the mechanical design's parameter which is extracted from the solid works. The electronics in this are power distribution board, protection board, safety kill and motor driver switch are major electronics involved in the UAGV $[18,19]$. The power distribution board and protection board protects the sensors and other logic circuits from high voltage and current. This also protects reverse polarity and isolation. The emergency and safety kill switches are of two ways, they are mechanical kill switch and remote kill switch. Kill switches are very important in emergency situation as the vehicle consists of up to 50 amps. The motor driver allows controlling the motor from the microcontroller [20, 21]. The operational diagram is given in Figure 3.

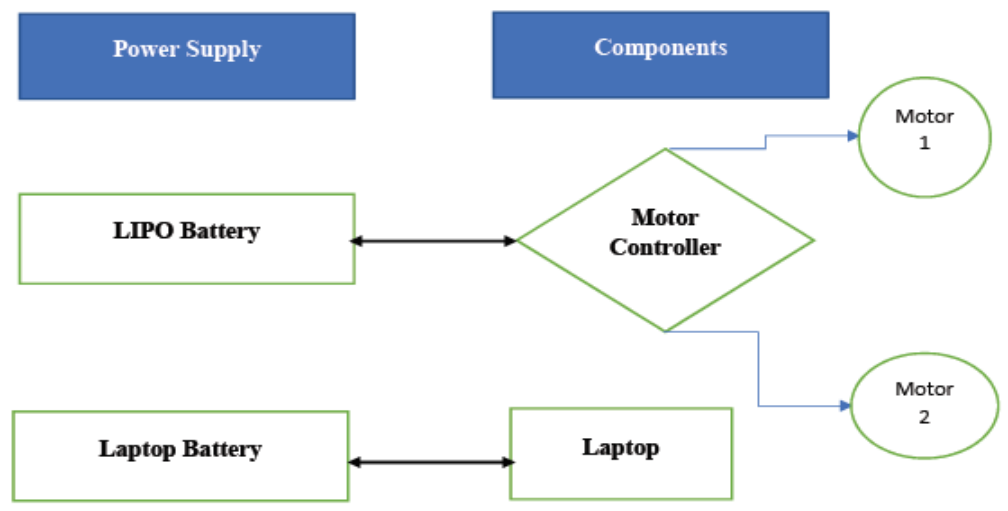

Figure 3. Operation of motor control

Image processing and computer vision plays a vital part in this autonomous navigation. This gives the complete map using camera and also detects the obstacle for the navigation and decision making purpose. Working principle of image processing and computer vision is shown in Figure 4. 


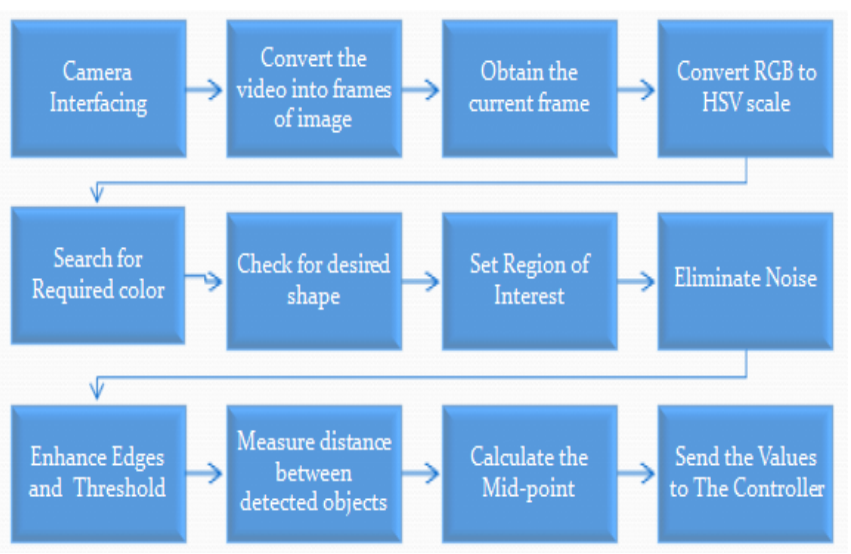

Figure 4. Working principle of image processing and computer vision

In this vision based navigation many algorithms are used. But the basic algorithm is as shown in the chart. In addition to this, human being is also detected for the autonomous navigation purpose, search and rescue. The artificial intelligence provides the thinking process to the vehicle, this make the vehicle smart and intelligent by choosing the shortest path from gps and vision based data for navigation by various algorithms such as Dijkstra's algorithm. The calculation of various parameters are given as follow as;

\subsection{Acceleration UAGV}

Acceleration of UGV on flat terrain probably wants the acceleration to be about half of maximum velocity. So, if say UAGV velocity is 3 feet per second and if the acceleration is around 1.5 feet per sq. second, then this would take 2 seconds to reach maximum speed.

\subsection{Force}

Force $=$ Mass $\times$ Acceleration

Torque $\times$ Rps $=($ Mass $\times$ Acceleration $\times$ velocity $\times 2 \pi) /$ Efficiency

Now,

UAGV total weight $=9 \mathrm{kgs}$

Desired velocity $=2 \mathrm{~m} / \mathrm{s}$

Desired acceleration $=1 \mathrm{~m} / \mathrm{sq}$. $\mathrm{sec}$

Expected efficiency $=75 \%$

Wheel Diameter $=0.215 \mathrm{~cm}$

Powered wheels $=2$

$$
\begin{aligned}
& =9 \times 2 \times 2 / 2 \pi \\
& =36 / 6.75 \\
& =11.5 \mathrm{Kg} \mathrm{M} \mathrm{Rps}
\end{aligned}
$$

\subsection{A super heavy duty DC gear motor of 200 RPM side shaft}

Rpm - 200 at 10 Volts

Voltage -4 to 12 Volts

Stall Torque $-47.19 \mathrm{Kg} \mathrm{Cm}$ at stalling current of $10.6 \mathrm{~A}$

Diameter of the shaft $-8 \mathrm{~mm}$

Length of the shaft -25 to $30 \mathrm{~mm}$

Assembly of the Gear - Spur

Type of Brush- carbon

Total weight -370 grams

So according to motor specification,

Torque $\times$ rps $=47.19 \times 3.33$

$=15.7 \mathrm{Kg}$ M Rps

Therefore

Torque $\times$ Rps $>$ (Mass $\times$ Acceleration $\times$ Velocity $) / 2 \pi$

Therefore $200 \mathrm{Rpm}$ heavy duty motor is sufficient for UAGV. 


\subsection{Runtime calculation for UAGV}

Battery used for two motor is $11.1 \mathrm{~V}, 2200 \mathrm{MAH}, 25 \mathrm{C}$, Zippy LipoBattery.

Now one motor requires 10.6 A at peak for $47.19 \mathrm{Kg}$ Cm Torque

Therefore

Maximum current drawn by 2 motors $=21.2 \mathrm{~A}$

The Ampere of the battery $=2200 \mathrm{MAH}=2.2 \mathrm{~A} / \mathrm{H}$

The Coulomb rating of the battery $=25 \mathrm{C}$

The maximum Discharge of the battery $=2.2 \times 25=55 \mathrm{~A}$

So the battery can provide sufficient power to both the motors.

The time the battery would take completely to drain at $2.2 \mathrm{~A}=60$ Mins

The time the Battery would take completely to drain at $21.2 \mathrm{~A}=2.2 \times 60 / 21.2=6.22 \mathrm{Mins}$

Connecting one more battery in parallel can give $=6.22 \times 2=12.44$ Mins.

\section{RESULTS AND DISCUSSION}

The UAGV operational results can be find by two modes:

Command Centre Control mode:

Based on the video record received from the on board camera located at the vehicle will be sent to the base station wirelessly. From the inputs, the reqired commands are sent to the UAGV remotely using wireless communication technologies.

Algorithm Design

User side

- The movement of the vehicle have been assingned with th Up Down Left and Right arrow keys.

- The input (pressed key) is converted as a special character to the Arduino device.

- The special characters sent will do the assigned function for them.

UAGV side

- The receiver unit in the UAGV monitors the input received from the special characters and also it acts as per the decision sent.

- Followed by the input the vehicle can move to the forward, reverse, left and right

- The forward and reverse operation uses a Clockwise and anticlockwise pin respectively.

- A Pulse Width Modulation (PWM) signal pin is assigned for for 80 - 120 degrees' turn. Finally, a d and H - Bridge is utilized for braking.

The graphical user interface (GUI) for common centre control mode is shown in Figure 5.

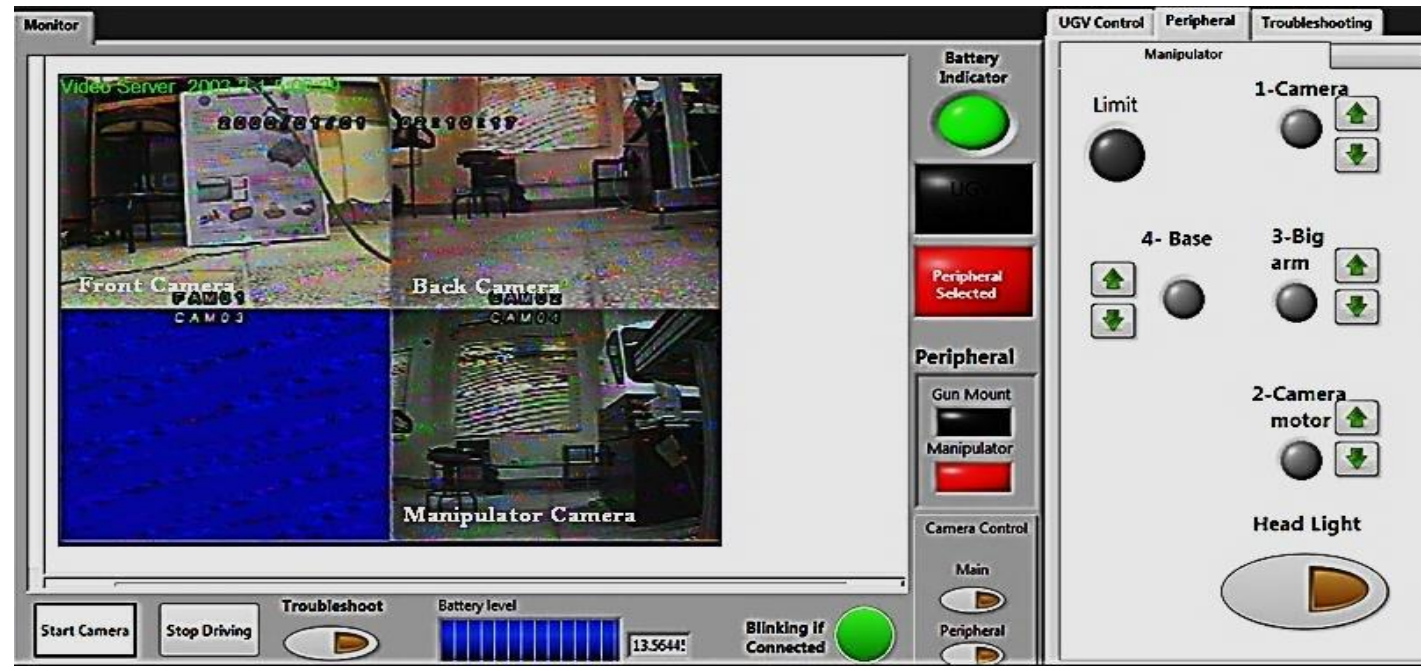

Figure 5. GUI of unmanned system 


\section{Autonomous Mode (Mode-2)}

Autonomous mode is capable of travelling from point A to point B without human navigation commands. Adjust strategies based on surroundings using obstacle detection algorithms. It enables an autonomous function with/without the human super vision. This operation can be accomplished by a navigation technology such as GPS.GUI of unmanned system of autonomous mode is given in Figure 6.

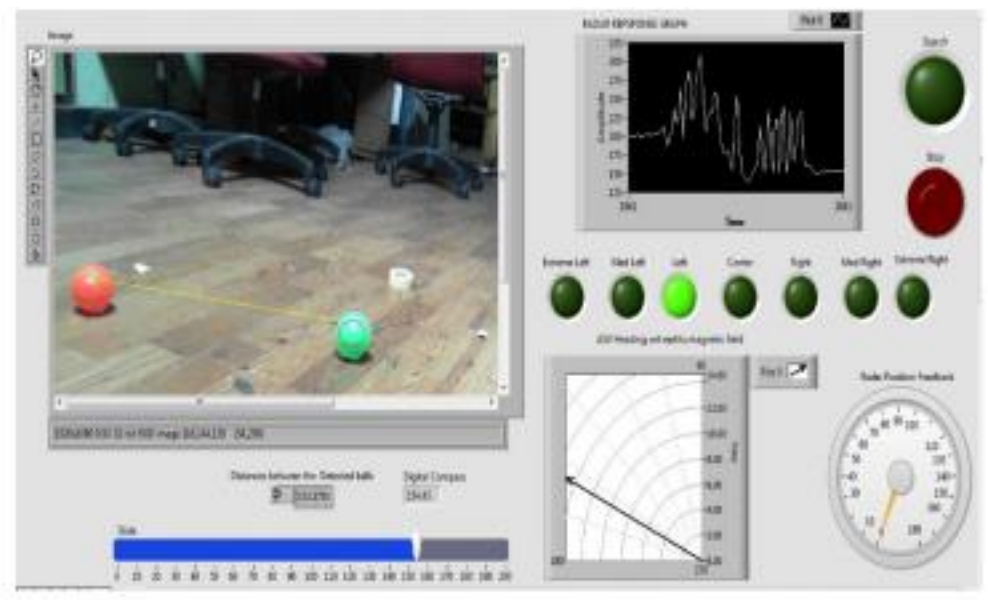

Figure 6. GUI of unmanned system autonomous mode

To control the movement of the vehicle, it is necessary to obtain the present GPS co-ordinates. The Compass located in the UAGV are used to acquire the data's for the user. By calculating the angles at which it orients with the desired direction by using a simple trigonometric functions. The path planning is based on the path finder algorithms such as shortest path algorithm. Meanwhile, Obstacle avoiding algorithm is incorporated to avoid obstacles while doing task. This can be done at hand in a most efficient manner based on the UR sensors values. The object detection and measuring the distance between the obstacle and the vehicle is given in the Figure 7 and Figure 8.

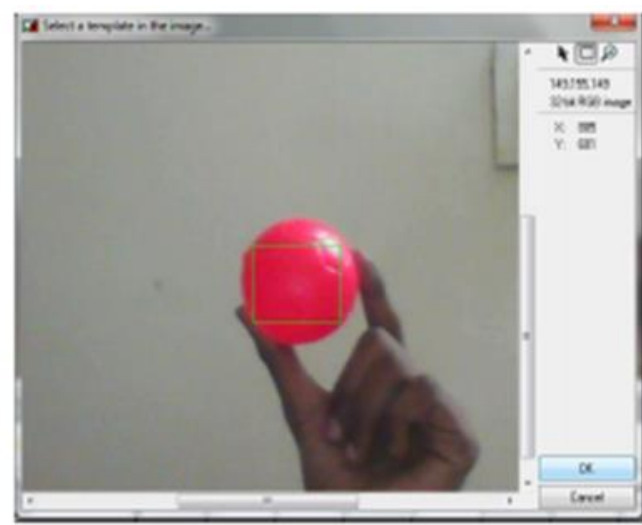

Figure 7. Object detection

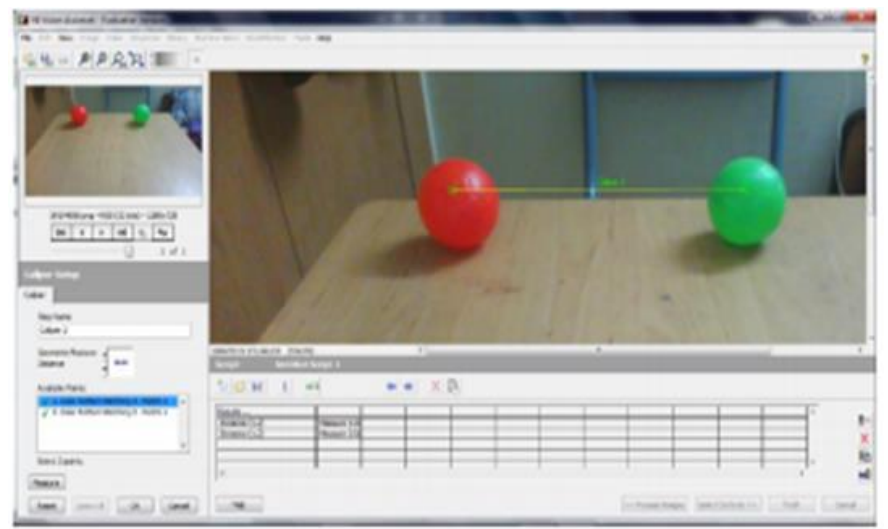

Figure 8. Distance between two objects using Image Processing

\section{CONCLUSION}

UAGV is a smart vehicle which is capable of driving without a driver. This can work during off and on road navigation. It can achieve the reduction of road accidents made by human error, and also used in military operation, carrying cargos, search, rescue etc reducing soldier's exposure to danger, freeing them to perform other duties. The operation is depending on the sensors to observe the environment also it reqires a high range of communication infrastructure. However, it can automatically take decisions on its own during any unpredictable situation. 


\section{REFERENCES}

[1]. L. H. Matthies, "Stereo vision for planetary rovers: stochastic modeling to near real-time implementation," International Journal of Computer VIsion, vol. 8(1), pp. 71-91, Jul 1992.

[2]. L. H. Matthies and P. Grandjean, "Stochastic performance modeling and evaluation of obstacle detectability with imaging range sensors," IEEE Transactions on Robotics and Automation, vol. 10(6), pp. 783-791, Dec 1994.

[3]. M. Drumheller and T. Poggio, "On parallel stereo," In Proc. IEEE Conf. on Robotics and Automation, IEEE, 1986, pp. 1439-1448.

[4]. "Artificial intelligence tutorials," edx.com

[5]. S. Kumar and P. Awasthi, "Navigation architecture for autonomous surveillance rover," 2012.

[6]. P. Z. Wenshuai Yu, Xuchu Yu and J. Zhoui, "A new framework of moving target detection and tracking for uav video application," 2004.

[7]. Sezer, C. Dikilita, Z. Ercan, H. Heceoglu, A. bner, A. Apak, M. Gokasan and A. Mugan, "Conversion of a conventional electric automobile into an unmanned ground vehicle (UGV)," Proceedings of the IEEE International Conference on Mechatronics, Apr 2012.

[8]. A. Mohebbi, S. Safaee, M. Keshmiri and S. Mohebbi, "Design, Simulation and Manufacturing of a Tracked Surveillance Unmanned Ground Vehicle," Proceedings of the IEEE International Conference on Robotics and Biomimetices, Dec 2010.

[9]. A. Bouhraoua, N. Merah, M. AlDajani and M. ElShafei, "Design and Implementation of an Unmanned Ground Vehicle for Security Applications," Proceedings of the 7th International Symposium on Mechatronics and its Applications, Apr 2010.

[10]. J. -H. Lim, S. -H. Song, J. -R. Son, T. -Y. Kuc, H. -S. Park and H. -S. Kim, “An Automated Test Method for Robot Platform and Its Components," International Journal of Software Engineering and Its Applications, vol. 4(3), 2010.

[11]. Shashidhar Muppidi, "Development of a Low Cost Controller and Navigation System for Unmanned Ground Vehicle," Master's Thesis, College of Engineering and Mineral Resources, West Virginia University 2008.

[12]. George Fernandez.S, K.Vijayakumar and R.Palanisamy, "GPS \& GSM Based Accident Detection and Auto Intimation," Indonesian Journal of Electrical Engineering and Computer Science (IJEECS), vol. 11(1), 2018.

[13]. Takashi Gomi, Koichi Ide, and Hirokazu Matsuo, "The Development of a Fully Autonomous Ground Vehicle (FAGV)," Applied AI Systems, Inc., Canada 1994.

[14].Cellini, M.; Mati, R.; Pollini, L.; Innocenti, M., "Obstacle Avoidance for Autonomous Ground Vehicles in Outdoor Environments," Intelligent Vehicles Symposium, 2007 IEEE, pp. 258-263, Jun 2007.

[15]. Reinhold Behringer, William Travis, Rob Daily, David Bevly, WilfriedKubinger, Wolfgang Herzner and Victor Fehlberg. "RASCAL - An Autonomous Ground Vehicle for Desert Driving in the DARPA Grand Challenge 2005," IEEE Conference on Intelligent Transportation Systems, 2005, pp. 644 - 649.

[16]. A. Ko and H. Y. K. Lau, "Robot Assisted Emergency Search and Rescue System With a Wireless Sensor Network," International Journal of Advanced Science and Technology, vol. 3, pp. 69-78, 2009.

[17]. Harmon S., "The Ground Surveillance Robot (GSR): An Autonomous Vehicle Designed to Transit Unknown Terrain," IEEE Journal of Robotics and Automation, vol. 3, no. 3 pp. 266-279, 1987.

[18].George Fernandez.S, K. Vijayakumar, D.Sattianadan, S. Vidyasagar, "Essential Need for Electric Vehicles and Infrastructure Advancement: Challenges in India," Indian Journal of Science and Technology, vol. 9(35), 2016.

[19].Hojjati-Emami, Khashayar, Dhillon, Balbir, Jenab, Kouroush, "Reliability prediction for the vehicles equipped with advanced driver assistance systems (ADAS) and passive safety systems (PSS)," Int. J. Indust. Eng. Comput, vol. 3(5), pp. 731-742, 2012.

[20]. Chris Hottentot, Veronique Meines, Mike Pinckaers, "Experiments on Autonomous and Automated Driving: An Overview 2015," ANWB, As of March 3, 2016.

[21]. Fagnant, Daniel J., Kockelman, Kara, "Preparing a nation for autonomous vehicles: opportunities, barriers and policy recommendations," Transport. Res. Part A: Policy Pract, vol.77, pp. 167-181, 2015. 